\title{
Entre amour et politique : métamorphoses ovidiennes à la fin du Moyen Âge
}

La fable de Céyx et Alcyoné, de l'Ovide moralisé à Christine de Pizan et Alain Chartier

\section{Jean-Claude Mühlethaler}

\section{CpenEdition}

\section{Journals}

Édition électronique

URL : https://journals.openedition.org/crm/76

DOI : $10.4000 / \mathrm{crm} .76$

ISSN : 1955-2424

Éditeur

Honoré Champion

Édition imprimée

Date de publication : 10 décembre 2002

ISSN : 1272-9752

Référence électronique

Jean-Claude Mühlethaler, «Entre amour et politique : métamorphoses ovidiennes à la fin du Moyen Âge », Cahiers de recherches médiévales [En ligne], 9 | 2002, mis en ligne le 04 septembre 2007, consulté le 15 décembre 2022. URL : http://journals.openedition.org/crm/76 ; DOI : https://doi.org/ $10.4000 / \mathrm{crm} .76$

Ce document a été généré automatiquement le 15 décembre 2022.

Tous droits réservés 


\section{Entre amour et politique : métamorphoses ovidiennes à la fin du Moyen Âge}

La fable de Céyx et Alcyoné, de l'Ovide moralisé à Christine de Pizan et Alain Chartier

Jean-Claude Mühlethaler

Alcyone war neben ihrem schlafenden Mann wie erstarrt. Nun

war sie allein mit den Bildern ihrer Angst. Und Cyparis

Projektor machte jedes dieser Bilder sichtbar, (...) ${ }^{1}$

1 Chaque époque a lu Ovide à la lumière de ses préoccupations, de sa vision du monde et de la littérature, dans un dialogue sans cesse renouvelé avec une œuvre riche et ouverte, dès le départ, à des interprétations divergentes ${ }^{2}$. Les auteurs ont trouvé dans les Métamophoses des métaphores ou des citations bienvenues, ils y ont pris des récits dont l'histoire ou les acteurs les interpellaient pour les récrire à leur gré. L'image d'Ovide s'est démultipliée au fil des rencontres individuelles et des siècles; son œuvre s'est transformée au contact de la liberté créatrice avec laquelle chaque écrivain se l'est appropriée. Les auteurs de la fin du Moyen Âge ne font pas exception, et l'influence de l'Ovide moralisé pèse moins lourd qu'on a bien voulu le croire et le dire. La réutilisation de la fable de Céyx et Alcyoné (Métam. XI, vv. 410-795) chez Guillaume de Machaut, Jean Froissart, Eustache Deschamps, Christine de Pizan et Alain Chartier laisse entrevoir, par delà certaines tendances générales, des possibilités surprenantes, ouvrant la voie à des récritures marquées du sceau de l'individualité. Déjà au XIV ${ }^{e}$ siècle, certains ont reconnu dans la matière ovidienne des "messages implicites " ${ }^{3}$, avec lesquels ils se sentent, plus personnellement, en consonance. Leur démarche annonce, de loin, celle qu'adopteront certains écrivains du $\mathrm{XX}^{\mathrm{e}}$ siècle qui ont découvert, chez Ovide, l'écho de leurs propres pensées et, avec Christoph Ransmayer (cité en épigraphe), la modernité de la fable et de la métamorphose.

2 Rappelons quelques faits indispensables à notre réflexion! Pour le public médiéval, l'Ovide moralisé est une translation des Métamorphoses. À l'exception d'ajouts, souvent 
importants ${ }^{4}$, il respecte l'ordre des fables et suit d'assez près sa source pour pouvoir être considéré, même par un lecteur moderne, comme une traduction. Le récit de Céyx et Alcyoné est d'une grande fidélité à l'hypotexte ${ }^{5}$. Il s'organise en deux parties: les vers 2996-3393 du livre XI ${ }^{6}$ racontent la mort de Céyx en mer, les vers 3394-3787 l'attente douloureuse d'Alcyoné, son rêve prophétique et sa métamorphose en oiseau, quand, à la vue du cadavre rapporté par les ondes, elle se jette à l'eau pour rejoindre son conjoint. S'y ajoute une glose dans laquelle, pour livrer au lecteur «de ces fables l'entendement» (v. 3789), le clerc applique au récit d'Ovide la démarche allégorique légitimée par saint Augustin dans le De civitate Dei (VII, 33) ${ }^{7}$. Il se distingue de ses prédécesseurs en combinant les différentes traditions d'exégèse ; l'expositio prend, chez lui, des dimensions inédites et elle propose au lecteur des approches dont la démarche peut varier d'une fable à l'autre, voire conduire à des interprétations contradictoires d'un seul et même récit ${ }^{8}$. L'unité des gloses ne repose guère que sur leur orientation générale, morale et chrétienne, par laquelle l'univers ovidien accède à un statut de vérité qui légitime les visées didactiques du clerc.

3 La fable de Céyx et Alcyoné est soumise à trois lectures qui, toutes, aboutissent à une seule et même leçon, celle du contemptus mundi. L'assimilation du voyage de Céyx, parti pour consulter l'oracle, à «ce pelerinage / Qui maine à la boneürté » (vv. 3809-3810), sert de point de départ. Dans cette perspective, chaque élément du navire (voile, ancre, câble, etc.) est pourvu d'un sens allégorique: il en vient ainsi à signifier le corps de l'homme, exposé à la mer de la vie, aux périls d'un «siecle» (v. 3848) trop sensible aux séductions du péché. Du niveau individuel, le clerc passe aux enjeux sociaux : l'image du navire ballotté par les flots représente aussi «Sainte Yglise» (v. 3960), guide des hommes sur le chemin du salut. La glose vire au blâme, car elle met en cause les «princes» et les «barons» (v. 4030) qui, loin de servir l'Église, refusent de lui obéir, la combattent en ne pensant qu'à leur profit personnel. Leur attitude s'explique par l'omniprésence de la «fraude» et de la «malice» (v. 4048), de la «glotonie» et de la "luxure» (v. 4060) dans un monde en plein déclin; elle témoigne aussi de l'effondrement des hiérarchies traditionnelles, et le clerc s'inquiète de la mise à mal de l'autorité spirituelle en des temps d'effervescence politique.

4 À l'époque de l'exil d'Avignon, après le conflit entre Philippe le Bel et Boniface VIII, puis celui qui opposa Jean XXII à l'empereur Louis de Bavière, l'Ovide moralisé introduit dans la glose un discours d'actualité dont on trouve les échos dans toute une série d'écrits, rédigés en français aussi bien qu'en latin au cours des premières décennies $d u$ $\mathrm{XIV}^{\mathrm{e}}$ siècle 9 . Le débat est européen : en France, Pierre Dubois, avocat à Coutances, rédige la Supplication du pueuble de France pour justifier la politique de Philippe le Bel, tandis que Gilles de Rome fournit des arguments au pape dans le De ecclesiastica potestate. L'autorité temporelle du Saint Siège est par contre niée par deux traités issus du milieu universitaire parisien: le Rex pacificus, puis le De regia potestate et papali (1302) de Jean Quidort plaident pour une limitation du droit d'ingérence du pape dans les affaires du royaume, car à leurs yeux seul le roi est responsable du bien commun. Les idées circulent, elles sont reprises et adaptées aux circonstances, adressées à d'autres princes: Dante écrit le De monarchia probablement à l'occasion de la descente d'Henri VII en Italie, Marsile de Padoue, auteur du Defensor pacis, est protégé par Louis de Bavière et, en 1339, Guillaume d'Ockham adresse une lettre (An princeps) à Edouard III d'Angleterre, dans laquelle il aborde la question de savoir si le roi peut disposer, sans 
l'autorisation du pape, des biens ecclésiastiques pour financer la guerre contre la France.

5 L'Ovide moralisé ne se limite pas à expliciter le sensus moralis caché sous le voile de la fable, il en suggère les implications référentielles. S'il pense, à l'instar des traités que nous venons d'évoquer, la politique en termes de morale ${ }^{10}$, il s'en distingue par le mouvement qui le conduit du récit mythologique à son allégorisation, puis à l'application de la glose à l'actualité du moment. Ce mouvement est celui même d'une satire comme le Roman de Fauvel (1310) qui se sert du récit allégorique (le triomphe du cheval fauve) pour dénoncer le renversement des valeurs à la cour de France. La parenté est plus profonde qu'on est tenté de le croire à une première lecture : les deux textes voient dans l'abaissement de l'Église et dans le triomphe des vices les signes avant-coureurs de la fin des temps. Ils trouvent, pour le dire, des mots, des rimes, qui se font écho :

\begin{tabular}{|l|l|}
\hline Saint Yglise flote et chancele. & (...) c'est une trop grande merveille \\
\hline \hline Se Diex ne garde sa nacele, & Que saint Pere ne s'apareille \\
\hline \hline Toute est descloëe et rompue. & De tost secourre a sa nacele, \\
\hline \hline (OM, vv. 4052-4054) & Qui si horriblement chancele. \\
\hline \hline & (Fauvel, vv. 371-374 $)$ \\
\hline
\end{tabular}

6 L'image du navire (en perdition) est trop courante au Moyen Âge, la rime «nacele » / «chancele» trop facile, pour conclure à l'influence du Roman de Fauvel sur l'Ovide moralisé- malgré leur utilisation dans des passages qui, chacun, dénoncent la déchéance morale de la société et les menaces qui pèsent sur l'Église. Et pourtant! Les deux textes ont circulé dans le même milieu, ils ont été l'un et l'autre illustrés par le même atelier parisien ${ }^{12}$ : que ce soit la version interpolée du Roman de Fauvel (BnF, fr. 146) ou le manuscrit de l'Ovide moralisé conservé à Rouen [ms. 1044 (0.4)], ils étaient destinés à des membres de la cour royale et de l'administration, intéressés, voire directement concernés par les bouleversements dans la sphère du pouvoir. La parenté des deux œuvres fait ressortir l'orientation satirique de la glose - et l'exemple n'est pas isolé! - dans l'Ovide moralisé. Elle révèle avec quelle facilité le message de la fable se laisse actualiser et récupérer, du moins ponctuellement, à des fins politiques. C'est là une démarche déjà récurrente dans les Bibles moralisées et illustrées du XIII ${ }^{\mathrm{e}}$ siècle $^{13}$ qui intègrent à la glose des attaques contre les ordres mendiants ou les Albigeois. Le commentaire moral n'est pas un but en soi ; il permet au clerc d'affirmer son savoir, son respect aussi des valeurs chrétiennes, de manière à asseoir son autorité face au public laïque. Sa supériorité morale et intellectuelle autorise les critiques qu'il adresse aux puissants, elle ouvre la porte à la dénonciation satirique, à la mise en cause de la société contemporaine.

7 Avec la troisième partie de la glose, le commentaire de l'Ovide moralisé se fait à nouveau général; les effets référentiels s'estompent, de sorte que disparait tout ancrage dans l'actualité politique du moment. La "sentence plus acordable» (v. 4118) au sens de la fable est une mise en garde contre la vanité des biens mondains, représentés par les vents, imprévisibles et dangereux, qu'Éole fait souffler à sa guise. Comme les oiseaux 
qui volent de ci, de là, «ces dames et ces damoisiaux» (v. 4137) risquent, quand ils s'adonnent à une vie de plaisirs, de s'y perdre corps et âme. L'allusion a beau être des plus fugitives, elle suffit à évoquer une jeunesse insouciante et, par-delà, le monde de la courtoisie... pour le rejeter, lui aussi, dans le domaine du péché.

De par son point de vue clérical, l'ovide moralisé ne pouvait guère être sensible aux virtualités courtoises des récits mythologiques, au contraire de Guillaume de Machaut qui se présente comme l'élève d'Amour et défend, dans son Prologue, une écriture consacrée à la louange des dames. Avec La Fontaine amoureuse (vers 1361), le poète champenois propose une relecture en clé courtoise de la fable de Céyx et Alcyoné... tout en se servant de l'Ovide moralisé. Les rencontres entre les deux textes sont assez nombreuses pour plaider en faveur d'une influence directe, malgré les différences qu'impose le passage de l'octosyllabe à rimes plates à l'hétérométrie et à la structure en miroir qui caractérise les cinquante strophes (10a10a10a4b10a10a10a4b / 10b10b10b4a10b10b10b4a) de la complainte du prince. Il y a des rimes communes :

\begin{tabular}{|l|l|}
\hline Pour la dolour qu'elle ot de son mari & Celui qui mors iere et peris, \\
\hline \hline Qui dedens mer par Fortune peri & Si li prie que ses maris \\
\hline \hline (Fontaine, vv. 556-557 $\left.7^{14}\right)$ & $($ OM, vv. 3412-3413) \\
\hline \hline & \\
\hline
\end{tabular}

9 Aux rimes communes s'ajoutent parfois des vers repris (presque) tels quels, comme cette présentation d'Iris, marquée du sceau de l'hypotexte ovidien. La «loial messagiere » rend le « fidissima nuntia » des Métamorphoses (XI, v. 585) :

\begin{tabular}{|l|l|}
\hline Juno qui vit et oï sa priere, & Ne pot Juno longues souffrir \\
\hline \hline Qui fu de cuer devote, humble et entiere, & Que cele perdist sa proiere. \\
\hline \hline Dist a Yris, sa loial messagiere: & Yrin, sa loial messagiere \\
\hline \hline (Fontaine, vv. 571-573) & Apele et aller li commande \\
\hline \hline & (OM, vv. 3423-3426) \\
\hline \hline & \\
\hline
\end{tabular}

Iris ne restera pas longtemps auprès du dieu du Sommeil, craignant d'être gagnée par la torpeur qui règne en ces lieux. Là encore, $L a$ Fontaine amoureuse fait en partie écho à l'ovide moralisé:

\begin{tabular}{|c|l|}
\hline Yris n'a pas atendut qu'il adjourne, & La messagiere atant s'en torne \\
\hline \hline Eins se depart et sans congié s'en tourne, & Qui ja se sent endeble et morne \\
\hline \hline Car volentiers ilec pas ne sejourne. & Pour le someil qui leenz iere. \\
\hline \hline Ce fist li lieus, & Si s'en est repairié ariere \\
\hline
\end{tabular}




\begin{tabular}{|l|l|}
\hline Qui la tenoit mate, endormie et mourne. & Par l'arc où elle estoir venue. \\
\hline \hline (Fontaine, v. 619-623) & (OM, v. 3510-3514) \\
\hline \hline & \\
\hline
\end{tabular}

11 Somnus, par contre, est rendu de manière variée : l'Ovide moralisé propose aussi bien « dieu de dormir ", « dieu dormant » que « diex de someil, diex de repos » (v. 3427, 3436, 3499), tandis que Guillaume de Machaut parle une fois du «dieu qui het noise et lumiere", l'autre du "dieus de sommeil» (Fontaine, v. 576 et 603). Les deux textes se rejoignent quand, à la suite d'Ovide, ils précisent qu'on n'entend ni cri de coq, ni aboiement de chien dans la demeure du dieu et qu'ils présentent les mille fils du Sommeil, les Songes, comme autant de maîtres de l'illusion et de la métamorphose :

\begin{tabular}{|c|l|}
\hline A leur voloir, car les fourmes prenoient & Li tiers prenoit d'iaue ou de fust \\
\hline \hline Des creatures & Et de toute autre creature \\
\hline \hline (.................................) & Qui ame n'a, forme et figure. \\
\hline \hline D'iaue, de feu, de toutes aventures, & (......................) \\
\hline \hline De fer, de fust, prenoient les figures. & C'est lor mestiers, c'est lor estudes \\
\hline \hline Autre mestier n'avoient, n'autres cures. & (OM, vv. 3535-3537 et 3545) \\
\hline \hline (Fontaine, vv. 637-638 et 647-649) & \\
\hline
\end{tabular}

12 Choisi pour annoncer la triste nouvelle à Alcyoné, Morphée «in faciem Ceycis abit » (Métam. XI, v. 653) :

\begin{tabular}{|c|l|}
\hline Lors Morpheüs & Si prent la forme qu'il savoit \\
\hline \hline Prist la fourme que Ceïs avoit nus & Que Ceys ot quant il iere vis. \\
\hline \hline Et moult forment fu mouilliez et emplus ; & Trop ot descoloré le vis, \\
\hline \hline Plus tors avoit les cheveus et locus & Si sambla mort et despoulliez. \\
\hline \hline C'une cordelle. & La barbe et les crins ot moulliez. \\
\hline \hline (Fontaine, vv. 658-662) & (OM, vv. 3563-3567) \\
\hline
\end{tabular}

Par delà une parenté indéniable, les différences entre les deux passages font ressortir la fidélité de l'Ovide moralisé aux Métamorphoses, au contraire de Guillaume de Machaut qui prend ses libertés. Le poète champenois ne translate pas, ne copie pas, il récrit en déplaçant les accents; la comparaison, inédite, avec la corde, en est la manifestation la plus frappante. On est loin de la démarche adoptée, à la fin de La Fontaine amoureuse, quand Machaut raconte le songe des cent sénateurs romains en s'appropriant des séquences entières de l'Ovide moralisé15. 
14 Avant tout, Guillaume de Machaut recentre le récit en faisant dès l'abord surgir l'image du noyé, avec la substitution significative de «nus» (v. 689) à la place de «vis» (OM, v. 3564). Le procédé est caractéristique du principe de condensation auquel est soumis l'ensemble de la fable : tout le voyage ainsi que le naufrage de Céyx sont évacués. Après la révélation de Morphée, Alcyoné est immédiatement métamorphosée en alcyon dans La Fontaine amoureuse (strophe XXIX) qui omet ainsi la longue plainte de l'épouse et la découverte macabre du corps de Céyx sur la grève. Chez Guillaume de Machaut, Alcyoné se retrouve au cœur du récit : oscillant entre la crainte et l'espoir, l'épouse est saisie dans sa douloureuse incertitude, à laquelle le songe révélateur met une fin.

La fable est évoquée au milieu de la complainte nocturne, au moment où le prince, désespéré, constate l'impossibilité de communiquer avec sa dame, que ce soit par écrit ou de vive voix : il lui «couvient autre voie querir» (v. 539) pour sortir de l'impasse! Le récit mythologique fonctionne comme un pivot grâce auquel le locuteur passe de la dysphorie initiale à l'euphorie finale, à cette «joie et deduit» (v. 926) que permet le retour d'Espérance, annoncé par l'envol de Céyx et d'Alcyoné métamorphosés en alcyons. Pour le prince, l'union finale affirme le triomphe de la vie et de l'amour sur la mort. La fable et le songe sont les mondes où le désir trouve son accomplissement.

L'hypotexte, inséré au cœur du discours amoureux, a un pouvoir régénérateur. Il sert de matrice à la seconde partie de la complainte qui l'interprète et le récrit en clé courtoise. Le prince déplace l'attention dont jouit Alcyoné dans le récit ovidien (tel qu'il l'a rappelé) vers Céyx, auquel il s'identifie par la souffrance: il supplie Morphée de révéler ses tourments à la dame afin de provoquer sa pitié. Le prince adapte le modèle à ses propos en plaçant sa propre prière à l'origine de l'intervention de Morphée, alors que le songe répondait, chez Ovide, à la requête d'Alcyoné. Selon une démarche héritée des trouvères, l'expression du désir est associée à la voix masculine, laquelle se substitue à la voix féminine du mythe. La dame ne prendra la parole qu'au moment de la réponse, quand, au cours du second songe, elle apparaîtra au poète et au prince, confirmant à ce dernier que lui seul occupe toutes ses pensées (vv. 2228-2234). Suite à quelques ajustements, quelques glissements subtils, l'épouse du roi antique cède la place à la dame courtoise.

17 Chez Guillaume de Machaut, la fable ouvre la voie au songe qui réussit là où ont échoué les plaintes, stériles, du prince enfermé dans sa douleur. Elle remplace une parole défaillante, incapable d'apporter à l'amant martyr la consolatio qu'il espère ; La Fontaine amoureuse se sert du récit de Céyx et Alcyoné de manière à offrir une nouvelle légitimité au discours courtois ${ }^{16}$, porteur d'espoir. Elle prend le contre-pied de l'Ovide moralisé dans la mesure où celui-ci condamne sans appel toute forme de plaisir au nom du contemptus mundi. Mais, placé sous le signe du jugement de Pâris et sous l'autorité de Vénus, à qui on doit aussi bien la destruction de Troie que l'apparition de la dame, le second rêve de La Fontaine amoureuse ne laisse pas d'inquiéter, car il comporte des failles difficilement compatibles avec l'exaltation de l'idéal courtois. Marqué par la tension entre deux visions contradictoires de l'amour, l'une conduisant au malheur de la communauté, l'autre au bonheur du couple, le message onirique est, pour le moins, ambigu.

18 Par sa «métaprogrammation $»^{17}$ du récit antique, Guillaume de Machaut révèle à quel point la fable ovidienne est riche d'interprétations virtuelles. La Fontaine amoureuse se distingue avant tout de l'Ovide moralisé par la subtilité ${ }^{18}$ d'une démarche faite 
d'interrogations et de doutes plutôt que d'interprétations claires et tranchées. Si affirmation il y a, c'est celle d'une écriture poétique, née d'une réflexion sur l'utilisation possible du mythe. Au songe d'Alcyoné répondent les songes parallèles du prince et du poète, de sorte que, de songe en songe, le texte entier finit par apparaître comme un seul et unique rêve: «Dites moy, fu ce bien songié ?» (v. 2848) est la question, emblématique, sur laquelle s'achève La Fontaine amoureuse.

Pour Guillaume de Machaut, l'écriture est songe, c'est-à-dire, dans un premier moment, évasion d'une réalité douloureuse. Elle est créatrice d'illusion, mais d'une illusion porteuse de vérité, ainsi que le rappelle en fin de parcours le rêve des cent sénateurs romains; en tant que telle, elle fonctionne comme une fable moderne qui, sous le voile de la fiction, recèle un sens caché. Machaut se veut l'Ovide de son temps, ce que l'auteur de l'Ovide moralisé n'a jamais recherché. Là où le clerc propose une récupération morale et chrétienne du texte antique par la glose, le poète champenois opte pour une récriture de la fable, laquelle apporte, du moins momentanément, au prince la consolation désirée.

Par la place privilégiée qu'elle accorde à la relation entre le prince et le poète, $L a$ Fontaine amoureuse se définit comme la rencontre entre deux subjectivités, comme une intimité partagée. C'est une écriture de rêve, fondamentalement euphorique, qui répond aux aléas de Fortune ${ }^{19}$ par la force de la fiction. Guillaume de Machaut prétend offrir une réponse à une douleur amoureuse née dans un contexte politique difficile, celui de la guerre de Cent Ans - à laquelle font une allusion voilée l'évocation des malheurs de Troie ainsi que le rêve apocalyptique des cent sénateurs romains. L'œuvre se veut, on le sait, un réconfort pour le prince Jean de Berry, sur le point de partir, captif, en Angleterre ; seulement, le discours courtois et le discours politique ne sont-ils pas contradictoire ${ }^{20}$ ?... En offrant au prince un échappatoire à son drame personnel, l'apparition de la dame le détourne des devoirs de sa charge, car, s'il apprend à prendre son mal en patience, il n'est pas amené à œuvrer au redressement de la France après le désastre de Poitiers. Le rêve, la littérature, n'invitent-ils pas à préférer le bien propre au bien commun - une solution difficilement compatible avec l'image que l'époque, imprégnée de pensée aristotélicienne, se fait habituellement du bon prince?

Quand Jean Froissart écrit Le Paradis d'Amour entre 1361 et 1362, il puise largement dans La Fontaine amoureuse ${ }^{21}$. Il en retient les aspects euphorique et onirique, mais évacue les implications politiques. Grâce à Plaisance et à Espérance, qui apparaissent après que l'acteur, "comme un homme desesperés» (v. 223), a exhalé sa douleur dans une complainte, il rencontre en rêve le dieu d'Amour ; fort de son autorité, celui-ci rétablit l'entente entre l'amant et sa dame. Froissart adopte ici un parcours narratif inspiré de La Fontaine amoureuse, mais il le corrige en attribuant le rôle de garants de la vérité courtoise à des personnifications plus dignes de confiance - Amour remplace Vénus ! que celles choisies par Machaut. Relevons surtout qu'il combine le cadre du songe, hérité du Roman de la Rose, et le souvenir du rêve prophétique d'Alcyoné :

Et non pour quant n'a pas lonch terme

Que de dormir euch voloir ferme,

Tant priai a Morpheüs,

A Juno et a Oleüs*, *Éole

Qu'il m'envoiierent les messages

De dormir.

(Paradis d'Amour, vv. 13-18) 
que, chez Machaut, la fable apparait au cœur de la complainte du prince, Froissart s'y réfère au début et à la fin du Paradis d'Amour, de manière à placer le récit tout entier sous l'égide du "noble dieu dormant» (v. 25). L'écriture est ici songe, et songe exclusivement: de la fable antique, Froissart ne retient que les noms des dieux (Morphée, Junon, Éole, Iris) dont l'intervention permet l'entrée en rêve, l'entrée en fiction. À aucun moment, il ne s'intéresse aux malheurs de Céyx ou d'Alcyoné ; il ne les évoque nulle part dans Le Paradis d'Amour, ne suggérant d'identification possible ni avec l'un, ni avec l'autre. Froissart se démarque ainsi de Machaut, et il s'en démarque d'autant plus qu'il réserve à Enclinpostair, fils inconnu du dieu du Sommeil, le rôle joué par Morphée dans La Fontaine amoureuse.

De manière générale, Froissart marginalise la mythologie antique dans son récit. Soit elle lui sert de comparant (Pygmalion, Achille, Narcisse, etc. ${ }^{22}$ ) pour dire la force de son amour, soit il énumère les amants et amantes du temps jadis, évoquant par exemple Hélène, Héro et Médée en compagnie des chevaliers de la Table ronde, de la châtelaine de Vergy, Guenièvre et Iseut (vv.988-993). Au contraire de Machaut, il ne propose aucun développement narratif, ni des fables héritées des Métamorphoses, ni de la matière de Troie. Tout se réduit chez Froissart à l'allusion, à d'infimes fragments mythologiques disséminés dans Le Paradis d'Amour, sorte d'arrière-fonds culturel sur lequel s'élève et triomphe son propre récit, célébration sans faille de l'idéal courtois. Il se sert de la mythologie comme d'un repoussoir pour mettre en valeur l'expérience de son moi onirique, et de lui seul ; il n'instaure pas, comme le fait La Fontaine amoureuse, un dialogue entre le poète et le prince.

Par la focalisation de l'intérêt sur son moi, par le traitement différent qu'il impose au matériau mythologique, Froissart se définit par opposition à Guillaume de Machaut. Le poète peut chanter son propre amour, car il est exemplaire autant que celui d'un prince ou que celui des amants les plus célèbres de la littérature antique et médiévale. Froissart n'éprouve aucun besoin de récrire une fable, de refaire le travail de l'Ovide moralisé... avec lequel un autre élève de Guillaume de Machaut, Eustache Deschamps, va renouer le dialogue, racontant à son tour l'histoire de Céyx et Alcyoné dans une ballade. Plusieurs rencontres au niveau de l'expression dans la première strophe, la présence d'Éole aussi, parlent en faveur d'un retour à l'ovide moralisé. La ballade s'ouvre pourtant sur un décasyllabe a minori dont le premier hémistiche fait écho au début de la fable dans La Fontaine amoureuse (v. 543: "Quant roy Ceïs»), tandis que le second hémistiche se présente comme la traduction d'un vers d'Ovide (Métam. XI, 412 : «ad Clarium parat ire deum ») :

\begin{tabular}{|l|l|}
\hline Quant roy Ceis ala au dieu de Claire & Ceys d'aller au dieu de Claire \\
\hline \hline Et se bouta es perilz de la mer, & \\
\hline \hline Pour enquerir aux sors tout son affaire & Por sort enquerre et por oïr \\
\hline \hline Alchione l'en voult souvent blamer & \\
\hline Pour les perilz, soy chetive clamer. & \\
\hline \hline Sa femme fut, d'Eolus fille estoit, & Pour Eolus, qui est mes peres, \\
\hline \hline Le dieu des vens, qui moult fait a doubter : & Et des vens rois et empereres \\
\hline
\end{tabular}


Foulz est li homs qui bon conseil ne croit.

\begin{tabular}{|l||l|}
\hline (ball. XXXV, vv. 1-8 & (OM, vv. 3004, 3006 et 3056-57) \\
\hline
\end{tabular}

Le refrain condense en un proverbe ${ }^{24}$ («Folz est li homs qui bon conseil ne croit») la leçon qu'Eustache Deschamps dégage du récit ovidien. Dans la première strophe, il s'en sert pour dénoncer l'aveuglement de Céyx; dans la seconde, Junon accède aux prières d'Alcyoné, non pas par pitié, mais pour démontrer la vérité de ce même proverbe qui fonctionne, mutatis mutandis, comme la glose dans l'ovide moralisé. Bien qu'il n'en adopte pas le point de vue chrétien, Eustache Deschamps reprend le rôle du clerc, quand il offre à son public une règle morale susceptible de s'adapter aux situations les plus diverses. Lui-même l'applique au domaine de l'amour en renouant, à la fin de la ballade, avec l'attitude qui fut celle du prince dans La Fontaine amoureuse. Convaincu par l'exemple de Céyx et d'Alcyoné, Deschamps se pose en lecteur avisé qui, ayant compris la leçon, adresse à son tour une prière à « saincte Juno » (v. 25). Que Morphée révèle les souffrances de l'amant - celles qu'il «n'ose n'escripre ne parler» (v. 26) - à la dame lointaine!

Dans la ballade, Eustache Deschamps tente de concilier, en passant par un proverbe passe-partout, la démarche de l'Ovide moralisé et celle de La Fontaine amoureuse. Le discours courtois apparait non pas comme un récit autonome, né des cendres de la fable dont il prendrait la place, mais comme l'illustration particulière d'une règle à laquelle il est soumis comme n'importe quel autre discours. Moraliste, Deschamps ne donne pas à la courtoisie le statut d'exception qu'elle a aux yeux de Guillaume de Machaut et, surtout, de Jean Froissart. L'idéal, chez lui, se fait terriblement quotidien; il est régi par les lois de la vie de tous les jours, il participe d'une vision pragmatique du monde - au point que le moi du poète n'a pas droit au rêve, car jamais la ballade ne lui ouvre les portes de l'espace onirique, porteur de consolation. Le discours s'interrompt au moment où l'amant adresse sa prière à Junon, dans l'attente d'une réponse qui, confinée dans le hors-texte, reste à venir.

À première vue, Christine de Pizan lit la fable de Céyx et Alcyoné dans la même perspective utilitaire que son contemporain Eustache Deschamps. La récriture en prose du récit ovidien est précédée, dans l'Epistre Othea, d'un quatrain, cette forme métrique que les auteurs de l'époque utilisaient volontiers à des fins didactiques :

Se par mer tu veulx entreprendre

Voyage perilleux et prendre,

Croy le conseil de Alchïoine,

De Ceÿs te dira l'essoine. ${ }^{25}$

Le refrain, chez Deschamps, ne disait pas autre chose : qu'on prête une oreille attentive aux bons conseils! Cette leçon trouve son pendant dans l'enseignement sur lequel débouche la fable et que Christine de Pizan clôt, à son habitude, par une citation attribuée à un auteur antique (Assaron, non identifié) : «Le sage s'efforce d'eslongner dommage, et le fol met peine a le trouver» (p. 311). L'exemplarité du récit est condensée ou, plutôt, réduite aux énoncés généraux qui l'encerclent.

Seulement, Christine de Pizan n'est pas Eustache Deschamps. Si elle évacue la possibilité d'une lecture courtoise de la fable, elle l'ouvre par contre à d'autres interprétations, conformes à son propre projet d'écriture. L'Espistre Othea est, on le sait, 
une missive envoyée par la déesse à Hector de Troie, âgé de quinze ans, afin de l'instruire. L'œuvre s'inscrit, comme bien d'autres écrits de Christine de Pizan ${ }^{26}$, dans la tradition des regimina principum, des miroirs des princes. Pour général qu'il soit, l'enseignement que la déesse tire de la fable ne s'adresse pas à n'importe quel homme, mais vise plus particulièrement «le bon chevalier» (p. 311) : il lui faut apprendre à écouter "ses bons amis", il doit s'entourer de conseillers fiables qui l'aident à faire ses choix. L'orientation politique du commentaire est confirmée par l'»allegorie»: l'homme dont l'esprit est «par male temptacion empesché» doit s'en remettre «a l'oppinion de l'Eglise» (p. 311). En affirmant la fonction de guide du pouvoir spirituel, Christine de Pizan partage une même vision du monde avec l'Ovide moralisé. Mais alors que le clerc reconnaissait l'Église dans le navire de Céyx ballotté par les flots (cf. supra), la poétesse fait d'Alcyoné la voix de la raison et, par delà, la voix de la vérité et de Dieu. Le changement d'éclairage, subtil, est significatif: Alcyoné se transforme en prophétesse, elle exerce auprès du roi, son époux, le rôle de médiatrice que Christine revendique volontiers pour la femme dans des écrits engagés comme Le Livre des trois Vertus ou l'Epistre a Isabeau de Baviere ${ }^{27}$.

Les enjeux politiques de l'«allegorie» s'imposent définitivement avec le rappel d'un exemple célèbre (cf. Gen. xLI), celui du pharaon dont Joseph sut gagner la confiance en lui expliquant ses songes : bien conseillé, le prince avait sauvé l'Égypte de la famine. En temps de crise, il faut savoir reconnaitre et écouter les voix prophétiques - telle est aussi l'opinion du Peuple, victime principale de la guerre dans Le Quadrilogue invectif d'Alain Chartier:

Si estoit la voix du peuple comme les mouetes qui par leur cry denoncent les floz de la mer, car noz parolles, que tu appelles murmure, signifioient des lors le meschief qui pour ces causes estoit a advenir. ${ }^{28}$

31 Ces mouettes qui annoncent la tempête imminente rappellent de près les alcyons. $\mathrm{Ne}$ vaut-il pas mieux, selon l'Ovide moralisé, éviter de s'embarquer, quand ils « vont voletant sor la marine» (v. 3780) ?... Guillaume de Machaut ne précise-t-il pas qu'ils sont annonciateurs de «fortune ou tempeste " (Fontaine amoureuse, v. 697)?... Bien que les Métamorphoses n'énoncent pas de règle météorologique à proprement parler, celle-ci est bien d'origine antique: elle se retrouve chez saint Ambroise (Hex. 5, 13, 41) et chez Isidore de Séville (Etym. XII, 7, 25). On ne saurait toutefois prouver que Le Quadrilogue invecitf s'insère précisément dans cette tradition, alors qu'il pourrait tout aussi bien avoir tiré profit d'une sagesse proverbiale, peut-être courante sur les côtes françaises ${ }^{29}$. L'ombre de l'Ovide moralisé et de La Fontaine amoureuse s'estompe ici ; le lien hypertextuel, lequel permet d'établir une filiation entre deux textes, cède la place à une transtextualité diffuse, source d'associations virtuelles, grâce auxquelles un lecteur (un clerc) cultivé établira une parenté entre des textes fort différents à première vue.

32 L'influence de l'ovide moralisé est d'autant moins probable que l'observation d'ordre météorologique se transforme, dans la bouche du Peuple, en énoncé politique. Confronté aux accusations du Chevalier qui dénonce son inconstance, il affirme la légitimité de ses plaintes. Vox populi, vox Dei ! C'est le Peuple qui est dans le vrai, car les nobles, affaiblis par leurs discordes et leur goût du luxe, n'ont pas assumé leur devoir face à l'occupant anglais. Paradoxalement, les implications politiques de la comparaison animalière renouent avec une certaine lecture (cf. supra) que l'ovide moralisé avait faite, non pas de la règle météorologique, mais du naufrage de Céyx. 
Christine de Pizan, nous l'avons vu, lui emboîte le pas, se démarquant de l'adaptation, par Guillaume de Machaut et Jean Froissart, de la fable à un contexte amoureux. Au XIV ${ }^{e}$ siècle, l'utilisation des Métamorphoses oscille entre deux tendances qui, chacune, laisse aux auteurs une marge de liberté assez grande pour choisir, puis réorienter à leur guise les éléments empruntés au mythe.

Il y a, d'un côté, le filon moral et politique (Ovide moralisé, Deschamps, Christine de Pizan); de l'autre, voici le filon courtois (Machaut, Froissart et, en partie, Deschamps). Remarquons que, cette fois encore ${ }^{30}$, l'ancrage référentiel du discours poétique s'impose avec force à l'époque de Charles VI, au moment où le royaume traverse une crise majeure. Il s'agit là d'un choix conscient puisque la fable de Céyx et d'Alcyoné offrait la possibilité de perpétuer la lecture courtoise, inaugurée par Guillaume de Machaut ; ni Christine de Pizan, ni Alain Chartier ne s'en sont souvenus, même pas dans leur lyrique d'amour.

Christine de Pizan recourt pourtant une seconde fois au même récit dans Le Livre de la Mutacion de Fortune, mais elle le récrit de manière inédite ${ }^{31}$ - au point que le passage ne se laisse que difficilement rattacher à l'un ou l'autre des filons. L'allusion se trouve à la fin de la première partie, au moment où l'acteur Christine voit le "patron de la nef " (son mari) disparaître dans la tourmente :

Dessoubz la pope, ou j'estoye,

Me lieve com femme enragiee,

Hault m'en monte et en mer plungiee

Me fusse, et ja n'y faillisse,

Qui ne me tenist, je y saillisse,

N'onc Alchyonie ne sailli

Plus tost en mer, quant lui failli

Ceÿs, que tant souloit amer ${ }^{32}$

Comme dans l'Epistre Othea, la relation entre Céyx et Alcyoné est ici placée sous le signe du bonheur et de la fidélité conjugales. Seulement, à la différence de la lettre envoyée au prince troyen, la Mutacion relit le récit d'Ovide en clé personnelle, d'un point de vue subjectif et féminin; le même mouvement de tendresse pousse Christine et Alcyoné à suivre l'époux défunt. La comparaison s'arrête là, car Christine se trouve sur le navire, prise elle aussi dans la tourmente. On l'empêche de se jeter à l'eau, et elle ne se métamorphosera pas en oiseau, mais en homme - comme Iphis au livre IX des Métamorphoses. Contrainte par Fortune de prendre le commandement du navire en perdition, elle se substitue au défunt, assumant des fonctions habituellement réservées aux hommes. Alcyoné sert ici de repoussoir ; son sort - enviable puisqu'elle rejoint son époux - ne fait que mieux ressortir la fatalité qui, à jamais, enferme Christine dans sa solitude de veuve, l'empêchant définitivement d'«estre femme» (v. 1399), de vivre selon les lois de Nature. La fin, radicalement différente, des deux récits peut se lire comme une rupture, voire un rejet ironique du modèle ovidien ${ }^{33}$ dont la clôture euphorique ne répond pas à l'expérience personnelle de la poétesse. Récit vain et plaisant, la fable est reléguée dans le domaine du rêve. Même la métamorphose d'Iphis véhicule l'illusion d'un bonheur désormais interdit à Christine, et l'intertexte (implicite) se fait ici l'expression d'un regret: devenue homme, Iphis n'avait-elle pas pu convoler en justes noces avec celle qu'elle aimait?... La fable, décidément, ne se récrit pas, elle ne s'inscrit pas dans le vécu. 

Pizan interprète sa propre vie et la découverte de sa vocation d'écrivaine ${ }^{34}$ à la lumière des Métamorphoses, les lectures proposées par la glose dans l'Ovide moralisé n'ont guère retenu son attention. Elle rompt ici avec une œuvre par rapport à laquelle se sont situés tous ceux - y compris elle-même dans l'Epistre Othea ! - qui ont (explicitement) utilisé la fable de Céyx et Alcyoné. Froissart, il est vrai, ne s'y rattache qu'indirectement, par le biais du dialogue qu'il instaure avec Guillaume de Machaut, mais Le Livre de la mutacion de Fortune n'en fait pas moins figure d'exception. Au début du XV ${ }^{\mathrm{e}}$ siècle, Christine de Pizan a trouvé dans la fable un instrument d'investigation du moi - et ceci en dehors de toute subjectivité courtoise. Elle ouvre la voie à une utilisation individualisée du mythe, plus proche de la liberté avec laquelle les auteurs modernes se sont appropriés Ovide.

Il n'y a plus guère de parenté entre le récit d'origine et la forme, tronquée et remodelée, sous laquelle il apparait dans la Mutacion de Fortune. La présence de la source s'y réduit à une allusion, et cette marginalisation de l'hypotexte conduit à un affaiblissement de son statut d'autorité, créant la plage de liberté nécessaire à l'éclosion d'une (r)écriture originale. La référence au monde ovidien permet à Christine de Pizan de penser sa propre métamorphose de femme en homme dans des circonstances difficiles; par une comparaison aux vagues relents mythiques, le Peuple, victime de la guerre, peut dire son indignation et son impuissance dans Le Quadrilogue invectif. L'évanescence, voire l'occultation de la source invitent à accorder une attention majeure aux réseaux transtextuels qui rapprochent des œuvres contemporaines, plutôt que de se fixer, dans un retour à la critique des sources, sur le seul lien entre l'hypertexte et un hypotexte qui, de surcroît, tend à s'effacer.

Jean Froissart, Christine de Pizan et Alain Chartier s'éloignent plus radicalement de la source que ne le font Guillaume de Machaut ou Eustache Deschamps. Si leurs œuvres se rencontrent, c'est qu'elles baignent, toutes, dans une même culture dont les traces incitent le lecteur à faire des rapprochements parfois inattendus, mais auxquels les auteurs eux-mêmes l'ont invité : n'ont-ils pas noué, par le biais de la fable, un dialogue (plus ou moins explicite) avec leurs contemporains ?... En récrivant ou en se détachant du récit transmis par l'Ovide moralisé, les auteurs se situent l'un par rapport à l'autre. Ils transforment la fable, en changent l'éclairage et le sens pour, en fin de compte, se démarquer et conquérir leur écriture individuelle. Ils confrontent le mythe, timidement chez Machaut, mais avec force sous la plume d'Alain Chartier et de Christine de Pizan, aux drames de l'Histoire, voire aux souffrances nées d'un vécu personnel.

\section{NOTES}

1.Christoph Ransmayr, Die letzte Welt, Nördlingen, F. Greno, 1988, p. 31. Dans ce roman, le récit de Céyx et Alcyoné, intégralement récrit, sert de point de départ à une quête d'Ovide sur les lieux mêmes de son exil : le monde antique et le monde contemporain renvoient l'un à l'autre, mais sans jamais se rejoindre complètement.

Cahiers de recherches médiévales, 9 | 2002 
2.Voir l'introduction de Ch. Martindale (éd.) à : Ovid Renewed. Ovidian Influences on Literature and Art from the Middle Ages to the Twentieth Century, Cambridge, University Press, 1988.

3.U. Heidmann Vischer, «Sed carmina maior imago sunt mea... Trois auteurs contemporains lisent Ovide : Vintila Horia, David Malouf et Christoph Ransmayr ", Nomen Latinum. Mélanges de langue, de littérature et de civilisation latines offerts au professeur André Schneider, éd. par D. Knoepfler, Neuchâtel-Genève, Faculté des Lettres - Droz, 1997, p. 486.

4.Cf. M.-R. Jung, «Aspects de l'Ovide moralisé », Ovidius redivivus. Von Ovid zu Dante, éd. par M. Picone et B. Zimmermann, Stuttgart, M und P, 1994, pp. 149-172.

5.Pour la terminologie, cf. G. Genette, Palimpsestes. La littérature au second degré, Paris, Seuil, 1982.

6.Nous citons d'après l'Ovide moralisé, poème du commencement du quatorzième siècle, publié d'après tous les manuscrits connus, vol. IV, éd. par C. de Boer, Wiesbaden, M. Sändig, 1967 (= Amsterdam, 1936). Nous le désignerons par le sigle OM.

7.Cf. P. Demats, Fabula. Trois études de mythographie antique et médiévale, Genève, Droz, 1973, p. 40.

8.Sur les innovations apportées par l'Ovide moralisé à la lecture des Métamorphoses, cf. R. Blumenfeld-Kosinski, Reading Myth. Classical Mythology and its Interpretations in Medieval French Literature, Stanford, University Press, 1997, chap. 3 ; A. Pairet, " Les Mutacions des fables »: figures de la métamorphose dans la littérature française du Moyen Âge, New Brunswick, Rutgers University, 2001 (version dactylographiée), chap. III : «Les Mutacions des fables dans l'Ovide moralisé».

9.Cf. S. Menaché, « La naissance d'une nouvelle source d'autorité : l'Université de Paris », Revue Historique, 544, 1982, pp. 305-327, et « Un peuple qui a sa demeure à part. Boniface VIII et le sentiment national français ", Francia, 12, 1984, pp. 193-208; E.H. Kantorowicz, Les deux corps du roi, trad. par J.-Ph. Genet et N. Genet, Paris, Gallimard, 1989, pp. 184-199 ; J.-C. Mühlethaler, Fauvel au pouvoir : lire la satire médiévale, Paris, Champion, 1994, pp. 290-303.

10.Le Moyen Âge (aristotélicien) a généralement pensé le politique en termes de morale. Cf. J. Blanchard et J.-C. Mühlethaler, Écriture et pouvoir à l'aube des temps modernes, Paris, PUF, 2002, pp. 7-14 et 33-36.

11.Gervais du Bus et Chaillou de Pestain, Roman de Fauvel, éd. par A. Långfors, trad. par M. Lecco, Milano, Luni, 1998, p. 104.

12.Sur le "Maître du Roman de Fauvel ", cf. l'introduction de François Avril à Le Roman de Fauvel in the Edition of Mesire Chaillou de Pesstain : A Reproduction in Facsimile of the Complete Manuscript, Paris, Bibliothèque Nationale, Fonds français 146, éd. par E. Roener, F. Avril et N.F. Regalado, New York, Broude Bros, 1990 ; A. Stones, « The Stylistic Context of the Roman de Fauvel, with a Note on Fauvain ", Fauvel Studies. Allegory, Chronicle, Music, and Image in Paris, Bibliothèque Nationale de France, MS Français 146, éd. par M. Bent et A. Wathey, Oxford, Clarendon Press, 1998, pp. 529-567.

13.Cf. R. Hausherr, "Sensus litteralis und sensus allegoricus in der Bible moralisée ", Frühmittelalterliche Studien, 6, 1972, pp. 356-380.

14. Nous citons d'après La Fontaine amoureuse, éd. et trad. par J. Cerquiglini, Paris, Stock, 1993. On lira avec profit l'introduction qui met en évidence les enjeux littéraires du texte de Machaut. 
15.Cf. R. Trachsler, «Cent Sénateurs, neuf soleils et un songe. Encore sur Machaut, la sibylle et le chaînon manquant ", Romania, 116, 1998, pp. 188-214.

16.Sur le lien entre mythe, amour et écriture, cf. R. Blumenfeld-Kosinski, Reading Myth, éd. cit., pp. 148-154 («Myth and Poetic Creation»), qui prolonge les réflexions de K. Brownlee, Poetic Identity in Guillaume de Machaut, Madison, University of Wisconsin Press, 1984, pp. 188-207 (consacrées à La Fontaine amoureuse).

17.Nous empruntons le terme à J. Ricardou, «Pour une théorie de la récriture ", Poétique, 77, 1989, pp. 3-15. Il désigne le fait de transformer un texte pour obtenir un but différent de celui poursuivi à l'origine.

18.Sur l'importance de cette notion dans l'art de Machaut, nous renvoyons à l'étude de J. Cerquiglini, «Un engin si soutil ». Guillaume de Machaut et l'écriture au XIVe siècle, Paris, Champion, 1985.

19.Sur le rôle de Fortune dans la version machaldienne de la fable, cf. H. Philipps, "Fortune and the Lady. Machaut, Chaucer and the Intertextual Dit ", Nottingham French Studies («Fortune and Women in Medieval Literature»), 38/2, 1999, pp. 126-127, qui rappelle aussi l'influence de Machaut sur Froissart.

20.Cf. M. J. Ehrhardt, « Machaut's Dit de la Fontaine amoureuse, the Choice of Paris and the Duties of Rulers ", Philological Quarterly, 59, 1980, pp. 119-139.

21.Le Paradis d'Amour, L'Orloge amoureus, éd. par P. F. Dembowski, Genève, Droz, 1986. L'éditeur signale l'influence de Machaut sur Froissart dans l'introduction (pp. 13, 26), mais les notes au texte ne fournissent aucune précision quant à la nature des emprunts. 22.Voir l'index des noms dans l'édition de P. F. Dembowski, pp. 128-132.

23. Fuvres complètes d'Eustache Deschamps, éd. par le marquis de Queux de Saint-Hilaire et G. Raynaud, Paris, F. Didot, 1878, vol. I, p. 118. Remarquons que les «perilz (de la mer) ", évoqués par deux fois, sont également récurrents dans la longue plainte d'Alcyoné (cf. Ovide moralisé, vv. 3031, 3037, 3049).

24.Cf. J. W. Hassell, Middle French Proverbs, Sentences, and Proverbial Phrases, Toronto, Pontifical Institute of Medaeval Studies, 1982, $\mathrm{n}^{\circ} \mathrm{C} 278$.

25. Epistre Othea, éd. par G. Parussa, Genève, Droz, 1999, p. 310 : «Texte .lxxix. ». Sur l'Ovide moralisé comme source de Christine de Pizan, voir l'introduction, pp. 32-36.

26.Cf. J.-C. Mühlethaler, «Traictier de vertu au proufit d'ordre de vivre : relire l'œuvre de Christine de Pizan à la lumière des miroirs des princes ", Contexts and Continuities. Proceedings of the IVth International Colloquium on Christine de Pizan, published in honour of Liliane Dulac, éd. par A. J. Kennedy, en collaboration avec R. Brown-Grant, J. C. Laidlaw et C. Müller, Glasgow, University Press, 2002, vol. 2, pp. 585-601.

27.Cf. J. Blanchard et J.-C. Mühlethaler, Écriture et pouvoir à l'aube des temps modernes, éd. cit., pp. 24-25 et 43-50.

28.Le Quadrilogue invectif, éd. par E. Droz, Paris, Champion, 1950 ( $2^{\mathrm{e}}$ édition revue), p. 38. 29. Mais on ne trouve rien dans les dictionnaires usuels ni dans les répertoires de proverbes et comparaisons proverbiales (G. Di Stefano, J. W. Hassell, J. Morawski, E. Schulze-Busacker). L'entrée «Möwe » chez W. Ziltener, Repertorium der Gleichnisse und bildhaften Vegleiche der okzitanischen und der französischen Versliteratur des Mittelalters, Heft 2 : «Natur - Zweiter Teil [Belebte Natur]», Berne, Francke, 1983, n 4436, relève seulement l'impossibilité de comparer la mouette à un oiseau de chasse.

30. Cf. J. Blanchard, «L'Entrée du poète dans le champ politique au XV $\mathrm{X}^{\mathrm{e}}$ siècle ", Annales E.S.C.. 1, 1986, pp 43-61 ; J.-C. Mühlethaler, «Le Tyran à table. Intertextualité et référence dans l'invective politique à l'époque de Charles VI ", Représentation, pouvoir et 
royauté, éd. par J. Blanchard, Paris, Picard, 1995, pp. 49-62, ainsi que : «Incipit Quadrilogium invectivum et comicum ad morum Gallicorum correctionem. Dalla definizione della satira all'uso degli animali nella letteratura impegnata ai tempi di Carlo VI : Alain Chartier e Eustache Deschamps ", L'Immagine Riflessa, N.S. VII/2, 1998 (Animali in letteratura), pp. 303-319.

31.Cf. R. Blumenfeld-Kosinski, Reading Myth, éd. cit., pp. 185-187.

32.Le Livre de la mutacion de Fortune, vol. I, éd. par S. Solente, Paris, Picard, 1959, vv. 1252-1259.

33.Cf. J. L. Kellogg, "Transforming Ovid. The Metamorphosis of Female Authority ", Christine de Pizan and the Categories of Difference, éd. par M. Desmond, MinneapolisLondon, University of Minnesota Press, 1998, surtout pp. 185-188.

34.Cf. K. Brownlee, «Ovide et le Moi poétique 'moderne' à la fin du Moyen Âge : Jean Froissart et Christine de Pizan ", Modernité du Moyen Âge. Le défi du passé, éd. par B. Cazelles et Ch. Méla, Genève, Droz, 1990, plus particulièrement pp. 162-170.

\section{AUTEUR}

JEAN-CLAUDE MÜHLETHALER

Universités de Lausanne et de Genève 